\title{
Facilitating Collaborative Engineering Analysis Problem Solving in Immer- sive Virtual Reality
}

\section{Alexander James Tuttle, University of Georgia}

Alexander Tuttle is an undergraduate student at the University of Georgia majoring in Computer Systems Engineering. He works in Dr. Kyle Johnsen's Virtual Experiences Laboratory where he develops and researches various Virtual Reality applications.

\section{Dr. Siddharth Savadatti, University of Georgia}

Dr. Siddharth Savadatti received his PhD in Computational Mechanics from North Carolina State University in 2011 and has since been on the faculty of the College of Engineering at the University of Georgia. He teaches mechanics and numerical methods courses such as Statics, Fluid Mechanics, Programming, Numerical Methods for Engineers and Finite Element Analysis. In addition to traditional face-to-face classes, he has designed and taught courses in fully online and completely flipped formats.

\section{Dr. Kyle Johnsen, University of Georgia}

Kyle Johnsen is an Associate Professor in the College of Engineering at the University of Georgia. Dr. Johnsen joined the University of Georgia in 2008 after earning his $\mathrm{PhD}$ in Computer Engineering from the University of Florida. His research focuses on emerging human-computer interaction technologies for health, education, and the environment. 


\section{Facilitating Collaborative Engineering Analysis Problem Solving in Immersive Virtual Reality}

\section{Introduction}

Though immersive virtual reality technology has existed for over half a century, early hardware was inaccessible for the vast majority of potential applications due to cost and issues that included significant discomfort and nausea [1]. Fortunately, the latest generations of immersive virtual reality equipment have largely mitigated many of these issues through more responsive sensing and display as well as the incorporating of several decades of usability research into application and interface design [2]. As a result, there has been a notable rise in the number of new applications being designed and tested, many of these aimed at addressing challenges and opportunities in education and training [3].

Within the education and training domain, the applications of immersive virtual reality have primarily leveraged the technology's capacity to engage learners in vibrant, realistic, interactive, to-scale environments. Examples include review and constructing 3D models (e.g. anatomy [4, 5], architecture [6]) and providing easy access to a variety of situations [7, 8], environments [9], objects/agents [10], and tools for practice $[11,12]$. Others have used virtual reality as a novel lecturing platform [13], exploiting the capacity of virtual reality to allow for the interactive illustration of 3D concepts. Similarly, this work aims to use virtual reality to facilitate students working together at a distance, on problems that better facilitate their conceptual understanding of engineering concepts.

The idea of using virtual worlds to collaborate remotely became popular in the late $2000 \mathrm{~s}$, the most notable general platform being Second Life [14]. Unfortunately, the platforms and technology of the time largely failed to deliver enough educational affordances [15]. Many of the challenges related to usability of the primary interface (keyboard and mouse) for 3D interaction. The steep learning curve of this interface for non-gamers makes it difficult for teachers, who may themselves be novices, to incorporate. Further, existing educational materials did not translate well or easily to the platform. Animated slides, for example, were difficult to incorporate, and whiteboards were not available [16]. These and other significant issues such as the ongoing cost of servers, though surmountable, have tempered the excitement about collaborative $3 \mathrm{D}$ virtual worlds in education.

Immersive virtual reality technology may reignite interest in collaborative 3D virtual worlds. While many of the same content issues exist today that existed a decade ago, the interface of immersive virtual reality is better suited to 3D interaction, particularly social interaction. Though users are often limited to controller and head-mounted-display movements to express themselves, these movements are mapped directly to hand and head movements, making their affordances natural. Moreover, head-mounted displays provide a stereoscopic 3D view, which allows users to make efficient distance and scale judgements in virtual worlds. In theory, by making the interface more natural, immersive virtual reality interfaces simplify working together in immersive virtual reality. 


\section{Purpose and Objective}

The overall purpose of this work was to leverage the affordances of immersive virtual reality for collaborative engineering problem solving in virtual reality. Towards this end, the following was built:

(a) Virtual Reality System for Collaborative Drawing

A virtual reality system which includes a hardware extension that uses a tracked graphics-tablet-pen for enhanced writing and drawing capabilities in immersive virtual reality

(b) Virtual Environment for Collaborative Engineering Problem Solving

An immersive virtual reality environment containing multiple whiteboards, a 3D angle and length measurement tool, and two typical engineering statics analysis problems

The research objective of this work was to determine if and how students engaged with and solved 3D engineering statics analysis problems presented through the graphics-tablet-enhanced immersive virtual reality platform. A pilot study involving 19 students from a local engineering statics course evaluated two key affordances of the system 1) taking measurements of important values in the problem, rather than having them provided, and 2) sharing the same virtual environment with another immersed user. These two affordances were expected to be related, in that students would work together to take measurements of the virtual objects and write them down; and, once measurements were taken, students were expected to work through the problem together using a set of virtual whiteboards that could be drawn on using the graphics tablets.

\section{Virtual Reality System for Collaborative Drawing}
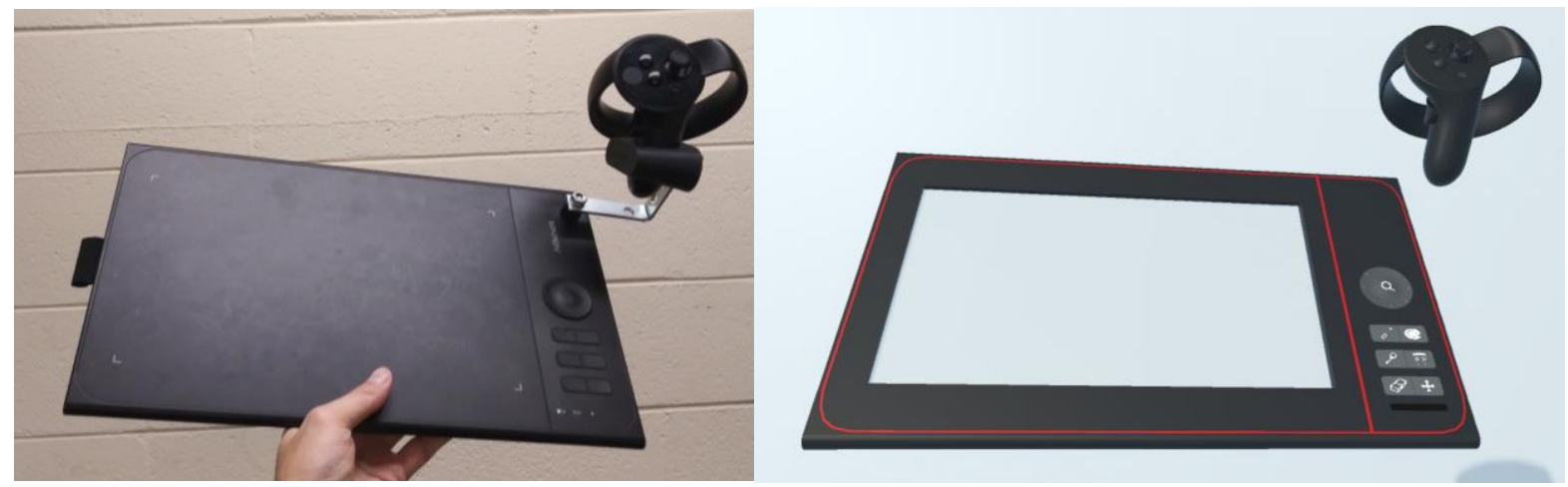

Figure 1 The physical (left) and virtual (right) tablets

In order to facilitate collaborative engineering problem solving in virtual reality, a hardware extension, tracked graphics tablets (pens), was introduced that were believed to be important to facilitate interaction. A graphics tablet facilitates high precision electronic drawing and writing and is a required tool for digital artists. By incorporating this tool, users could draw diagrams and take notes in educational social encounters in virtual reality. Though it is possible to draw in mid-air using the controllers, this is more fatiguing and imprecise relative to using a pen and having a writing surface. A graphics tablet, by contrast, is designed to be comfortable to use for prolonged writing and drawing, activities that are commonplace while solving engineering problems. 
The base hardware platform for the system was a popular offthe-shelf virtual reality system from Oculus Virtual Reality. This system provided a wired head-mounted display (Oculus Rift CV1) and two wireless controllers (Oculus Touch), each of which contains embedded sensors and lights that are viewed by a set of surrounding infrared cameras. The system was further augmented with an off-the-shelf wireless graphics tablet and pen (XP-Pen Star 06), to which one of the controllers was attached with a friction-mount as shown in Figure 1 (left). A "Virtual-Reality-Ready" laptop (Alienware 15 R3, Intel Core i7 CPU, Nvidia GeForce 1070 GPU, 16GB RAM) was connected to these devices to process user input and was also used to simulate and render the virtual environment.

A single controller was attached to the tablet for three primary reasons. First, as the tablet itself had no pose tracking system, this rigid adapter could be used to infer the tablet's pose from

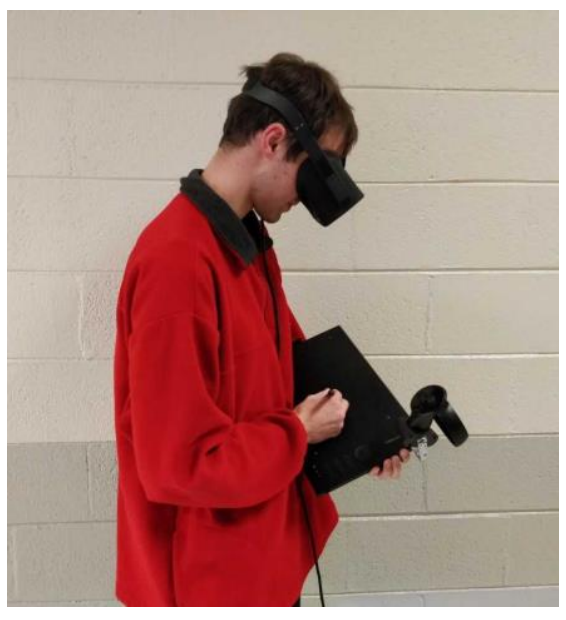

Figure 2 A user wearing the head-mounted display while drawing on a graphics tablet in virtual reality. the controller's pose based on a known affine transformation between the two devices. This information allowed a virtual version of the tablet to be rendered in the virtual environment as shown in Figure 1 (right). Second, the adapter allowed the controller to be removed as needed, to be used independently from the tablet. This action could be performed quickly, without looking at the tablet, due to its distinctive shape. This was important because, when the controller was removed, the tablet visualization would no longer move. When docking and undocking, the pen could be attached to its metal bracket using a magnet affixed to the pen. Finally, the second controller was not used so that the user could easily hold the tablet and pen while moving around. Only a single wire connected the user to the laptop (see Figure 2), which is now optional due to the availability of wireless display adapters.

The Unity3D game engine was used to generate the immersive virtual environment and provide the user interface, which had three primary modes of interaction. The first two are commonly found in virtual reality systems, consisting of a way to move around the virtual environment (when the physical environment is smaller than the virtual environment) and a way to manipulate objects. To move, the user could push in a direction on the controller's thumb-joystick, which slowly (to reduce simulator sickness) progressed the user in that direction along the floor plane. To manipulate virtual objects, the user could grab them by intersecting the virtual controller with their surface, hold the controller trigger, which then attached the two until the trigger was released in a different location.

The last interaction mode, drawing, was performed by manipulating the graphics tablet and pen. The tablet consisted of a digitizer surface, 6 buttons, and a rotary-wheel. To

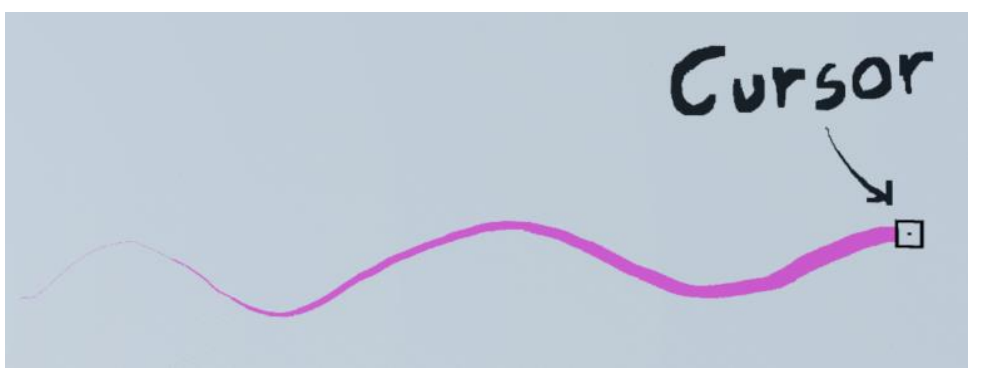

Figure 3 A line drawn at varying width. The cursor indicates the current location of the pen tip. 
draw, the user would bring the pen tip near the digitizer surface. This had to be done largely by proprioception and feel, since the user could not see the pen within the virtual reality interface. However, once near the tablet (within about $3 \mathrm{~cm}$ ), the digitizer would detect the pen tip and send the 2D location, enabling the system to show a cursor, not unlike a mouse cursor, that facilitated precise pen tip placement. When pressed against the surface, the digitizer would send the location as well as the tip pressure (8,192 levels), enabling the system to begin drawing a virtual line, which would continue at varying thickness according to the pressure exerted as the pen was dragged, as depicted in Figure 3.

The buttons on the tablet, seen in Figure 1, offered further functionalities. Color was determined by pressing the palette button, and then selecting a color from a continuous grid with the pen tip. Similarly, the eyedropper button changed the color to any pixel selected within the drawing. The eraser button selectively erased pixels by changing the pen color to the current background color. The user could clear the whole drawing by holding down the trash can button for several seconds, a time that reduced the risk of unintentional erasure. Beyond these standard drawing facilities, the user could scale the drawing using the click-wheel or move the drawing by pressing the arrows button and then dragging with the pen. These features allowed the digitizer's physical surface $(50,000 \times 30,000,5000$ lines per physical inch) to be mapped to a larger virtual area. The last button, the laser pointer, had two purposes. By holding it, a red laser line would emerge from the tablet's front. This could be used as an indicator or to select distant objects.

Most importantly, the system supported collaboration across all interaction modes by as many users as the network and computer hardware can support (three simultaneous users have been tested, but more are possible). Each user could see, in real time, exactly what the other users were drawing, the other users' avatars, tablets, and laser lines (Figure $4 \& 6$ ), the other users manipulate objects in the scene, and could communicate with the other user using a voice-overinternet-protocol service. This was all supported across the internet, allowing collaborators to be in geographically distant locations.

\section{Virtual Environment for Collaborative Engineering Analysis Problem Solving}

Beyond the facilities described above, a specialized virtual environment and user interface were created to support collaborative engineering analysis and problem solving. As seen in Figure 5, this environment imitated a small classroom with an array of three large whiteboards on the front wall. Users could draw to the whiteboards by using the graphics

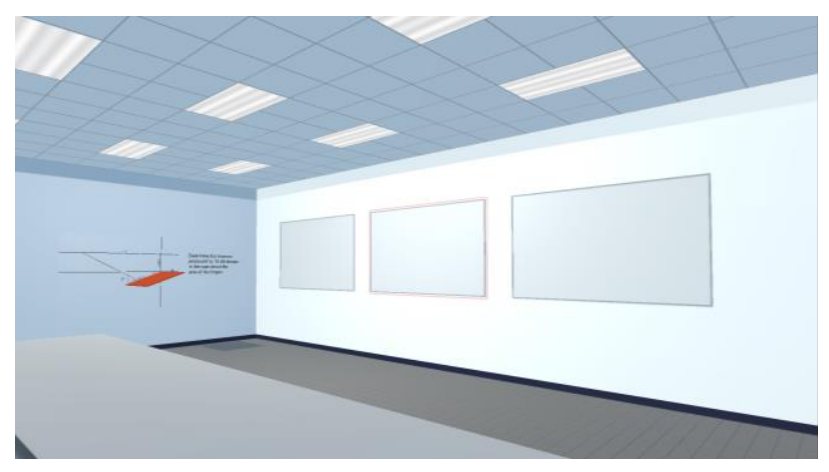

Figure 5 The collaborative engineering analysis environment. 
tablet, which would mirror the drawing to a selected whiteboard. The whiteboard was selected by with the laser pointer, which would immediately cause the tablet to mirror the selected whiteboard, as depicted in Figure 6. Three whiteboards were used, as opposed to having one large whiteboard because the graphics tablet has a fixed physical aspect ratio. Using the same aspect ratio whiteboards made maximal use of the tablet's drawing surface without non-uniform scaling of the image on either surface. The

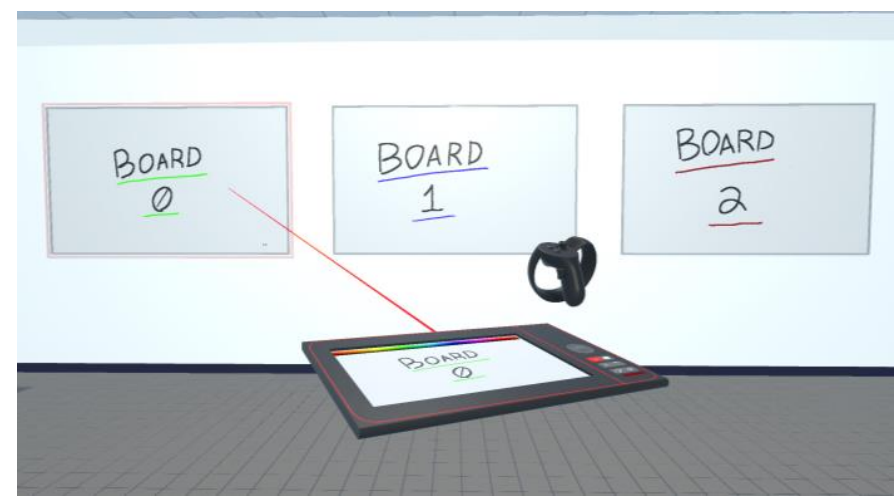

Figure $6 \mathrm{~A}$ virtual laser pointer selects the active whiteboard. educational purpose of these whiteboards was to provide ample room for drawing and writing, facilitating social, embodied discussions to take place about these artifacts. Though the tablets could also be used for the same purpose, the larger size of the whiteboards, like in a classroom, were easier to use for group discussion.

To make further use of the immersive virtual reality system beyond collaborative drawing, 3D problems were incorporated into the virtual environment. In addition to 3D graphics, these problems contained textual descriptions and annotations, such as coordinate systems and measurements, much like textbook problems. However, because they were contained within immersive virtual reality, they could be viewed from any reachable perspective (unlike a problem in a traditional textbook). Moreover, the stereoscopic rendering within the headmounted display meant that spatial judgements could be natural. Using the collaborative features, students could have discussions about the problems, referencing specific parts of the 3D structure.

As problems were being constructed, it was recognized that the measurements that are typically provided to students may be unnecessary inside virtual reality. As the problems are physically reachable, it would be possible for students to take their own measurements if they were provided the tools to do so. The logic was that analyzing real-world problems nearly always requires physical measurement as a first step. Skipping measurements could thus be detrimental to student's later ability to solve problems in practice.

To support taking typical measurements, a specialized virtual tool, shown in Figure 7 , was added to the virtual environment.

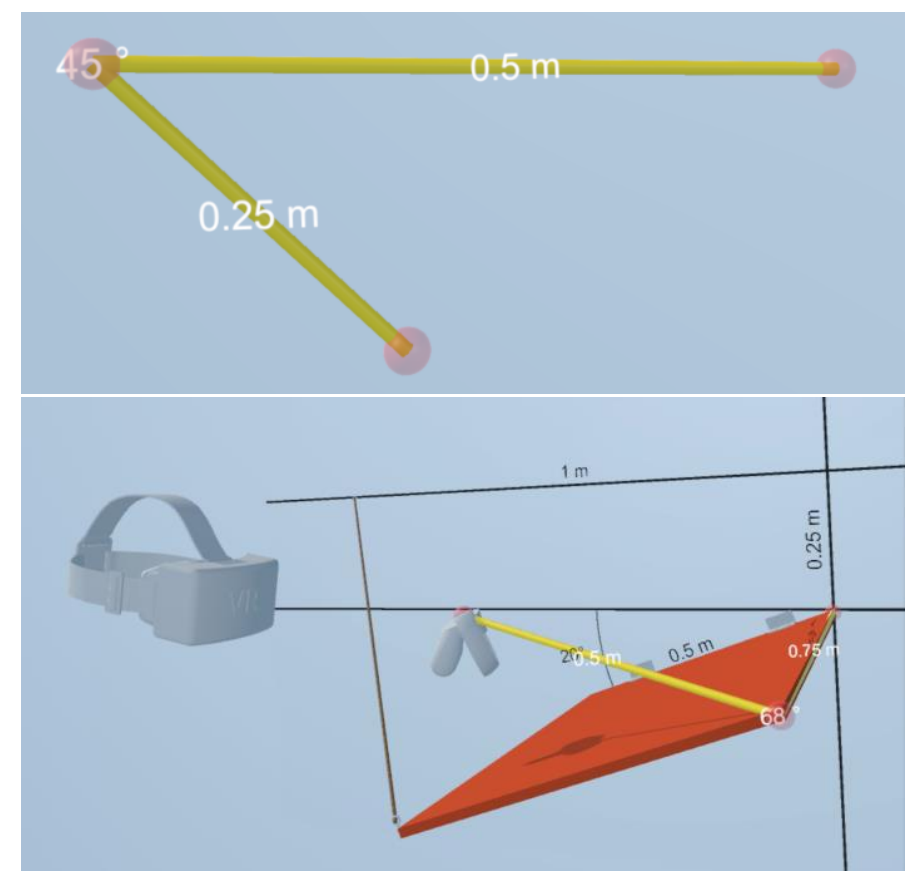

Figure 7 The 3D angle and length measurement tool in use. 
The tool, which was effectively a combination of a tape-measure and protractor, consisted of two variable length rods that were joined at one end. The tool was manipulated by taking the controller out of its dock and grabbing the nodes (shown in red) with the controller and dragging them to a new position. Grabbing an end-node only moved that node, but grabbing the rods moved the entire tool. These facilities allowed the bars to be adjusted together to take length and angle measurements. Though this meant that the measurements taken by students would likely be imperfect, it further emphasized real-world practice, where measurements would vary. It added realistic uncertainty to problems in an efficient, non-arbitrary way.

The final feature of the environment was that all activity was recorded, including the position and rotation of all devices, measuring tools, pen-strokes and buttons pressed. This deep-logging is expected to be increasingly useful as a means for students and instructors to reflect on past performance, and potentially to automatically analyze real-time problem-solving behavior. It can also be used as an efficient way to watch a recorded lecture from an arbitrary viewing perspective, as was proposed in [13]. The whiteboard diagrams and writing could also be saved as artifacts of the analysis.

\section{Pilot Study}

Though presented above as a sequential process, in truth, the hardware, user interface, and application were developed together, largely in parallel from an initial idea that collaboration and drawing were important for real-world engineering problem solving, but they were largely absent elements in virtual reality systems which inhibited the adoption of virtual reality systems into education of all sorts. Engineering statics was adopted as a target application because of its strong reliance on the analysis of 3D structures, which required significant drawing and writing, potentially benefiting from collaboration. Measurement was later added as another potential benefit that is more exclusive to engineering education. Better understanding these two features were the target of the following pilot study.

5.1 Study Objectives - Qualifying the interaction between students, the engineering analysis problems presented in virtual reality, and the user interface were the primary objectives of the pilot study. To increase the authenticity and viability of the study, it was also proposed to involve students currently taking an engineering statics course as participants, who would be expected to have the training already to solve engineering statics problems presented in a traditional form. The major questions were thus about the virtual reality system's usability in solving problems together. Of concern were the novelty of the virtual reality interface alongside the simultaneous novelty of the problem presentation and collaborative strategy.

5.2 Physical Environment and Population - Students enrolled in a local engineering statics course at the University of Georgia were recruited to participate in the study, incentivized by a \$20 (USD) gift card. After recruitment, students were matched in pairs with similar engineering analysis skill levels (based on the statics instructor's recommendation) and availability. In total, 19 students, 8 pairs and 3 individuals, participated in the study over the course of three days ( 2 days before, and the day of, the final examination for the course). 
The study took place within a virtual reality laboratory, where the equipment was available, and which was quiet during finals week. Two identical virtual reality systems were used during collaborative sessions, which were directly networked through an ethernet connection (1Gbps) to eliminate the possibility of any network disruptions. As the participants were physically colocated, voice communication was disabled to eliminate audio conflicts. The tracking spaces did not overlap within the laboratory environment so that participants would not physically contact each other. Generally, though, participants used the joystick to move around the virtual classroom environment rather than physically walking.

5.3 Variables - Two independent variables were under consideration. The first was measurement, where participants would either be given enough measurements to solve the problems or would be required to take their own measurements using the virtual 3D angle and length measurement tool. Originally, this was intended to be the only independent variable in the study, but a consequence of scheduling participants in pairs was that some could not be matched. As a result, this added another dimension to the study (collaborative vs. alone).

Two similar (and typical) engineering statics problems were chosen for this study. Termed the 'hinge' and 'anvil' problems (Figure 8), they each involved determining the moment produced by a force about a specified axis on a 3D structure. Each of the 11 sessions (8 collaborative, 3 individual) involved solving both problems. For each session, one of the two problems had its measurements visible, while the other had them hidden to force the participant(s) to make use of the virtual measurement tool. Between the order of solving the problems (first and second) and the availability of measurements (visible and hidden), there were four possible permutations for any given session. The number of instances of each permutation were evened out as much as possible given the small sample size.

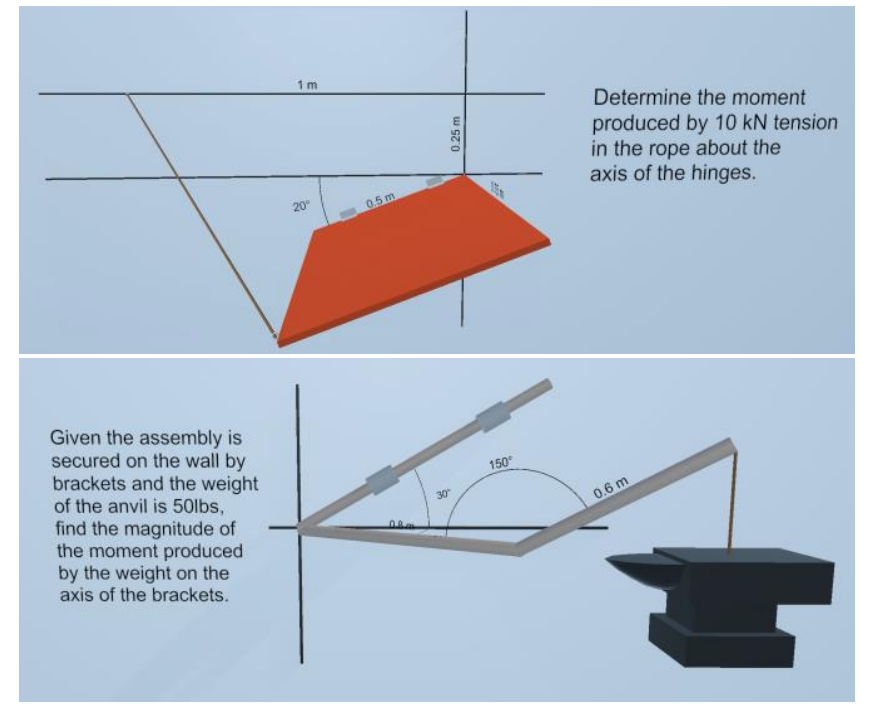

Figure 8 The two engineering statics analysis problems included in the study; the 'hinge' (top) and 'anvil' (bottom) problems.

5.4 Measures - Several measures were used for each session. A background survey provided self-reported age and gender as well as experience and skill with games and virtual reality, each of which could qualify results. In addition, commonly used surveys relating to the psychological constructs of presence [17] and copresence [18] were used to quantify how immersive the experience was for participants. For usability and difficulty, the NASA TLX inventory [19] was used. Performance was measured by saving the session's drawings to an image and these images were graded by the statics instructor. If the participants consented to it, voice recordings were taken to be able to reflect on how well the groups worked together. Finally, notes were taken by the observer and a debriefing survey with comments was administered to the subjects. 
5.5 Procedures - After consent was determined, each participant filled out a short background survey. Then the participants were briefed on the purpose of the experiment and what they would be doing. Next, the participants were taught how to use the system before being given roughly five minutes to practice. After this, each participant was given roughly 15-20 minutes to solve the first problem, and then another 15-20 minutes to solve the second problem. The time range was due to participants being "close" to a solution and allowing them to finish. While solving the problems, the experimenter observed and took general notes on anything notable that happened. Once the problems were completed, the rest of the forms were given and completed. Lastly, the participants gave comments on the system and its potential as an alternate way of learning.

\section{Results}

All 19 participants completed the experiment in 11 sessions (8 pairs and 3 individuals). Sessions were randomly distributed to all four permutations. Though the number of available participants was limited, this distribution served to reduce the risk of bias towards one configuration when comparing visible and hidden measurements. Unfortunately, for one group the software crashed towards the end of their second problem, and one group erased some of their work before it could be saved, resulting in a null datapoint for that problem.

Sessions were similar in terms of participants' background. There were equal numbers of male and female participants, and these were distributed randomly across variable permutations and between sessions. Only one participant had a moderate level of prior virtual reality experience, with the remainder having low or none, and only 2 participants reported above average 3D gaming experience.

Participants working individually reported an overall higher sense of presence in the virtual reality environment, as determined by the number of items on the scale marked as 6 or higher than those working in pairs [17]. All 3 individuals had a score of 4 or higher, while only 2 out of 16 working in pairs did. However, co-presence (the sense of being with others) was generally high in the paired sessions. This may be a consequence of participants being in the same physical room as their partner, which may have reduced their individual sense of presence.

NASA TLX scores (all out of 21) were consistent with expectations that the task was relatively difficult, with some notable differences between the participants working alone and those working in pairs. Individuals rated the experience as requiring higher effort (17.0 vs 13.9$)$ but were generally less frustrated (6.7 vs. 10.9). The overall level of

\begin{tabular}{|r|r|r|}
\hline & Individuals & Pairs \\
\hline Effort & 17 & 13.9 \\
\hline Frustration & 6.7 & 10.9 \\
\hline Physical Demand & 5 & 6.5 \\
\hline Mental Demand & 15 & 14 \\
\hline
\end{tabular}

Table 1 Mean NASA TLX Subscores by group condition (Each score is out of 21) 
Also confirming the difficulty of the task, the average score for the 'hinge' problem was $56 \%$ and for the 'anvil' problem it was $45 \%$. In addition, of the 22 instances of problem solving $(2$ problems per session with 11 sessions), 12 instances were stopped by the researchers before the

\begin{tabular}{|r|r|r|r|}
\hline & Individuals & Pairs & Combined \\
\hline $\begin{array}{r}\text { Hinge } \\
\text { Problem }\end{array}$ & $74 \%$ & $50 \%$ & $57 \%$ \\
\hline Anvil Problem & $57 \%$ & $42 \%$ & $45 \%$ \\
\hline Totals & $67 \%$ & $44 \%$ & $51 \%$ \\
\hline
\end{tabular}

Table 2 Mean scores by problem and condition. participants felt they had completed solving the problem. Researchers stopped these instances because the planned 15-20 min were exhausted. Scores between problems were correlated at a medium level $\left(\mathrm{R}^{2}=0.59\right)$. On an average, participants working alone received higher scores than those working in pairs (67\% vs. $44 \%$ ) and the first problem solved in each session received lower scores than the second ( $45 \%$ vs. $57 \%$ ).

\section{Discussion}

The statics instructor who graded the solutions to the two problems (and from whose engineering statics course the participants were recruited), commented on the quality of these solutions relative to those typically received as a regular part of his course. Compared to solutions to similar problems on paper, the work shown in virtual reality was less systematic and lacked logical presentation. However, participants did seem to have understood the problem and what was asked of them, and in general identified the concepts that needed to be applied. The instructor was worried that students may not have been able to relate the immersive 3D representation to the typical textbook representation that students were familiar with, but this seemed to not have been the case. The instructor felt that the scores were somewhat lower than what would have been expected on similar test problems in his course, but cited several reasons this may have been the case, including that (a) students are generally more prepared for tests, (b) time provided may not have been enough, (c) it is not typical to provide problems without measurements, and (d) students are unfamiliar with virtual reality as a medium of problem presentation and solving.

The data and participant comments supported several of these statements. Many participants provided statements that supported visualizing the problem in three dimensions, commenting that this benefitted their understanding of the problem, e.g. the "3D model was nice to tour" and "being able to see 3D models is great." However, some groups were not convinced that the 3D space supported the solving of these problems, suggesting that the 3D space was too "abstract" and that there were "too many options for converting to $2 \mathrm{D}$ (where to put the origin, what measurements to take, etc.)". Several participants stated that as all the 3D problems in their textbook/course were presented on 2D paper, when tasked with a problem visualized in 3D space it just meant they had to convert it to a 2D diagram before solving it. The groups that felt this way also noted that if they were taught with $3 \mathrm{D}$ depictions in the first place, this negative aspect would be bypassed. This tension is highlighted by one participant who commented that "the benefits of $3 \mathrm{D}$ viewing are better than the downside of having to convert to a $2 \mathrm{D}$ picture." 
The average solution time was 20.6 min when measurements were hidden and 19.7 min when measurements were visible, though performance with and without measurements was similar $(53 \%$ visible, $51 \%$ hidden). Participant comments were revealing. When asked which option they

\begin{tabular}{|r|r|r|}
\hline & Hidden & Visible \\
\hline Solution Time & $20.6 \mathrm{~min}$ & $19.7 \mathrm{~min}$ \\
\hline Solution Score & $51 \%$ & $53 \%$ \\
\hline
\end{tabular}

Table 3 Participant performance with measurements hidden and visible. preferred (visible or hidden measurements) 6 preferred having measurements given (visible), while 3 preferred taking measurements themselves (hidden). Participants had insightful comments, such as "when there are no measurements the problem becomes harder since given measurements often hint towards the right way of solving the problem", "Measuring gave a better sense of the problem", and "doing measurements on your own is simpler since less trig is needed".

The novelty of the medium was also likely a factor in performance. As participant performance generally improved between the first and second problem, more practice may improve usability. Several participants commented on challenges with the user interface, most commonly highlighting the difficulty of writing without seeing the pen, although several commented that this became less difficult over time. Several participants expressed frustration with the measuring tool, commenting that the task of measuring was "annoying", and that they wanted the tool to have options such as "snap points" for moving nodes to important parts of the model and "axis constraints" that locked movement to a specific plane or axis. Unfortunately, participants in four sessions also reported temporary negative health effects (dizziness, headache, and nausea), which were not unexpected, given the length of time they were immersed and inexperience with virtual reality, with most attributing this problem to moving around the environment using the joystick.

Concerning collaboration, participants had few comments, though they were generally positive. The experimenter's notes were more revealing. Of the eight pairs, all worked together to take measurements, as this meant that one person could measure, while the other wrote down measurements. After taking measurements, the majority performed calculations independently, providing two solutions, qualifying the erratic solutions observed by the instructor, who was intentionally kept unaware of the number of participants providing the solution.

\section{Conclusions and Future Work}

This work demonstrated how immersive virtual reality systems could be designed for engineering students to analyze systems and solve problems. Students were given typical textbook problems in the engineering statics domain, but they were provided with a 3D system instead of a 2D diagram. Using a virtual tool, students were able to take length and angle measurements of the 3D system instead of having them always provided. In addition, a graphics tablet allowed students to take notes and to write down complete solutions on virtual whiteboards provided alongside the problems. Finally, students could share the virtual environment and whiteboards, allowing for collaborative problem solving.

A pilot study of 19 engineering students who were enrolled in an engineering statics course demonstrated that the system was usable, either individually or in pairs. Students quickly 
became familiar with the interface, despite the majority having had no prior experience using immersive virtual reality. Students spent most of the time engaged in solving the problems. After touring the problem together and taking measurements, pairs tended to work independently, despite being allowed to solve the problem together. Users were highly engaged with the system, and many positive comments were received about the idea, with little push-back on using the system in practice, except that students would have liked to see the system used for lecturing prior to them having to use it to solve problems.

Currently, the main limitation is still the hardware required to use the system effectively, as well as the physical space and setup of the system. Fortunately, there are several next-generation virtual reality devices headed to the market that will be self-contained, greatly decreasing the expense of the system, though connecting the graphics tablet with these devices may be a challenge. For now, the best use case for the system would likely be within online engineering courses, where all students could conceivably have or rent a system. For in-person courses, the system could be used during lecture by the instructor, small-group discussions by students, or office hours. Future work will continue to advance the system, turning it into a platform for engineering education across a range of subjects. There is great interest in pairing the analysis capabilities with more elaborate 3D environments that showcase the potential to display systems of any scale, beyond the current room-scale systems. In addition, allowing these systems to be dynamic simulations provides even more didactic possibilities. Ultimately, the goal is to use the capabilities of these systems to move beyond the confines of classrooms and current remote education systems, whenever desired, to broaden our understanding of how students learn to bridge the gap between engineering theory and practical application.

\section{Acknowledgements}

Anton Franzluebbers provided support during the user study as well as the 3D models for the virtual room and tablet.

[1] S. V. G. Cobb, S. Nichols, A. Ramsey, and J. R. Wilson, "Virtual reality-induced symptoms and effects (VRISE)," Presence: Teleoperators \& Virtual Environments, vol. 8, no. 2, pp. 169-186, 1999.

[2] P. Ziegler, D. Roth, A. Knots, M. Kreuzer, and S. von Mammen, "Simulator Sick but still Immersed: A Comparison of Head-Object Collision Handling and their Impact on Fun, Immersion, and Simulator Sickness," in 2018 IEEE Conference on Virtual Reality and $3 D$ User Interfaces (VR), 2018, pp. 743-744: IEEE.

[3] D. Velev and P. Zlateva, "Virtual reality challenges in education and training," International Journal of Learning and Teaching, vol. 3, no. 1, pp. 33-37, 2017.

[4] J. H. Seo et al., "Anatomy builder VR: applying a constructive learning method in the virtual reality canine skeletal system," in International Conference on Applied Human Factors and Ergonomics, 2017, pp. 245-252: Springer.

[5] S. Marks, D. White, and M. Singh, "Getting up your nose: a virtual reality education tool for nasal cavity anatomy," in SIGGRAPH Asia 2017 Symposium on Education, 2017, p. 1: ACM.

[6] T. Hilfert and M. König, "Low-cost virtual reality environment for engineering and construction," Visualization in Engineering, vol. 4, no. 1, p. 2, 2016. 
[7] J. Kawai, H. Mitsuhara, and M. Shishibori, "Game-based evacuation drill using augmented reality and head-mounted display," Interactive Technology and Smart Education, vol. 13, no. 3, pp. 186-201, 2016.

[8] C. Li, W. Liang, C. Quigley, Y. Zhao, and L.-F. Yu, "Earthquake safety training through virtual drills," IEEE transactions on visualization and computer graphics, vol. 23, no. 4, pp. 1275-1284, 2017.

[9] E. Hu-Au and J. J. Lee, "Virtual reality in education: a tool for learning in the experience age," International Journal of Innovation in Education, vol. 4, no. 4, pp. 215-226, 2017.

[10] K. Johnsen and B. Lok, "An Evaluation of Immersive Displays for Virtual Human Experiences," presented at the IEEE Virtual Reality, 2008.

[11] J. Bertrand, A. Bhargava, K. C. Madathil, A. Gramopadhye, and S. V. Babu, "The effects of presentation method and simulation fidelity on psychomotor education in a bimanual metrology training simulation," in 3D User Interfaces (3DUI), 2017 IEEE Symposium on, 2017, pp. 59-68: IEEE.

[12] A. Bhargava, J. W. Bertrand, A. K. Gramopadhye, K. C. Madathil, and S. V. Babu, "Evaluating Multiple Levels of an Interaction Fidelity Continuum on Performance and Learning in Near-Field Training Simulations," IEEE transactions on visualization and computer graphics, vol. 24, no. 4, pp. 1418-1427, 2018.

[13] M. Melatti and K. Johnsen, "Virtual Reality mediated instruction and learning," in $K-12$ Embodied Learning through Virtual \& Augmented Reality (KELVAR), 2017 IEEE Virtual Reality Workshop on, 2017, pp. 1-6: IEEE.

[14] C. Wankel and J. Kingsley, Higher education in virtual worlds: Teaching and learning in Second Life. Emerald Group Publishing, 2009.

[15] K. Andreas, T. Tsiatsos, T. Terzidou, and A. Pomportsis, "Fostering collaborative learning in Second Life: Metaphors and affordances," Computers \& Education, vol. 55, no. 2, pp. 603-615, 2010.

[16] C. Atkins and M. Caukill, "Serious fun and serious learning: The challenge of Second Life," Learning and teaching in the virtual world of Second Life, pp. 79-89, 2009.

[17] M. Usoh, E. Catena, S. Arman, and M. Slater, "Using Presence Questionnaires in Reality," Presence: Teleoperators \& Virtual Environments, vol. 9, no. 5, pp. 497-503, 2000.

[18] J. N. Bailenson, K. Swinth, C. Hoyt, S. Persky, A. Dimov, and J. Blascovich, "The independent and interactive effects of embodied-agent appearance and behavior on selfreport, cognitive, and behavioral markers of copresence in immersive virutal environments," Presence: Teleoperators \& Virtual Environments, vol. 14, pp. 379-393, 2005.

[19] S. G. Hart and L. E. Staveland, "Development of NASA-TLX (Task Load Index): Results of empirical and theoretical research," in Advances in psychology, vol. 52: Elsevier, 1988, pp. 139-183. 